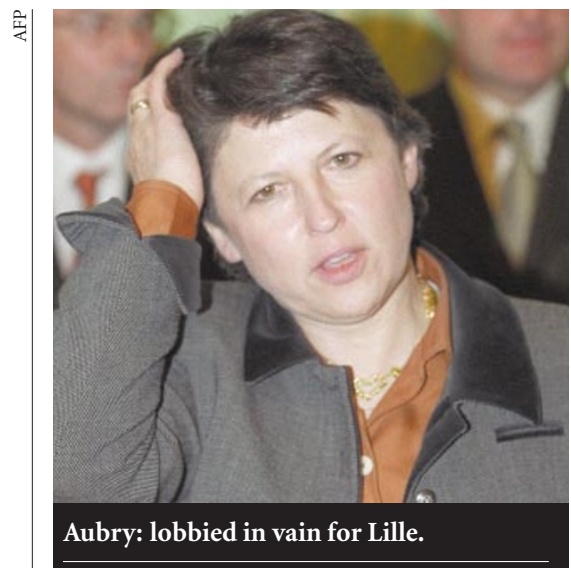

Allègre, who revealed few details regarding his decisions on Soleil, Schwarzenberg made the report public.

Taking a swipe at his predecessor, who announced Soleil's abandonment during August, when France is on holiday, Schwarzenberg said he had waited until September to "to avoid any announcement in the month of August".

The regions will provide an unprecedented proportion of Soleil's costs; the state will end up paying just FFr200 million-300 million. The regions already contribute towards the cost of the planned national university, and Schwarzenberg hinted that regional money would play an increasing role in the research budget.

The machine will have an energy range of 2.5 to $2.75 \mathrm{GeV}$, producing beams from the ultraviolet to both soft and hard X-rays. Four beamlines will be dedicated to biological research instead of the two proposed originally. Twentyfour beamlines will be built at first, increasing to 40 .

Soleil will have a company's legal structure, enabling it to function continually and offer higher salaries: rules at public research organizations would not allow such flexibility.

\title{
Drug-resistant HIV shows a worrying increase in the UK
}

Natasha Loder, London

The United Kingdom has seen a large increase in new cases of multidrug-resistant HIV over the past five years, according to a researcher at the Public Health Laboratory Service (PHLS) who is calling for wider testing for resistance.

Multidrug-resistant HIV is resistant to one or more members of all three classes of anti-HIV drug. It develops in patients who are receiving therapy, and is transmitted by normal HIV infection routes. This is the first time such an increase has been seen.

Deenan Pillay, of the PHLS's Antiviral Susceptibility Reference Unit at the University of Birmingham, presented the figures at last week's British Association science festival at Imperial College in London. The data are from a register of patients who had recently contracted HIV, all testing HIVnegative in the preceding 18 months or showing recent seroconversion at the time.

Pillay found that five out of 24 patients who had been infected up to May 2000 had key resistance mutations. In the previous five years, only one in 40 was similarly infected.

The news will disappoint many scientists and clinicians. High-level resistance mutations are less prevalent in studies in other countries, leading some to hope they would remain rare. Robert Shafer, an assistant professor of medicine at the University of Stanford, California, is cautious about interpreting unpublished figures.

But he says that "if correct, they are important and distressing," because they suggest that it is not just those unaware of their infection who are spreading HIV, but also people who know of their condition and have access to treatment.

Resistance often develops because

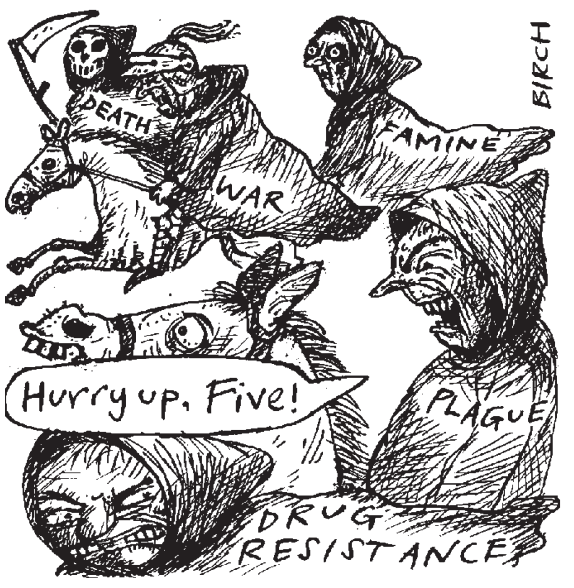

patients cannot maintain the harsh drugtaking regime. But even those with full compliance have a $20 \%$ failure rate in the United Kingdom, possibly because the treatment is unable to halt viral replication entirely.

Pillay told the meeting that "the emergence of drug resistance is virtually inevitable" and warned that the spread of resistant strains in the United Kingdom threatened "the success we have seen in antiviral therapy to date". New classes of drug could offer hope to patients with multidrugresistant HIV.

There are currently 14 anti-HIV drugs in three classes: nucleoside analogue reversetranscriptase inhibitors, non-nucleoside reverse-transcriptase inhibitors, and protease inhibitors. Despite rising numbers of HIV cases, the number of deaths from AIDS in England and Wales has halved since 1995 - partly owing to the introduction of protease inhibitors in 1996 as part of combination therapies that suppress viral replication.

Once a virus develops resistance this often confers high- or low-level resistance to other drugs in the same class. But occasionally a mutation can make the virus particularly sensitive to another drug in the same class.

Shafer has created a database linking data on the sequences of HIV-1 reverse transcriptase and protease, drug treatment histories and drug susceptibility, which he hopes will help in the design of treatment regimes. Genetic tests can show why patients are failing therapy and could help doctors design the best long-term drug treatment regime.

Pillay, whose unit conducts such tests, says this "is now recommended in order to guide the most effective therapies". But each

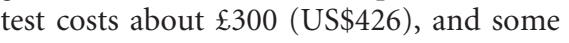
National Health Service trusts are refusing to pay for them. Shafer says that preliminary data suggest that the cost of testing are offset by a decreased use of ineffective drugs. 